\title{
On time-reversibility of linear stochastic models *
}

\author{
Tryphon T. Georgiou* and Anders Lindquist ** \\ * Department of Electrical $\&$ Computer Engineering \\ University of Minnesota, Minneapolis, Minnesota \\ tryphon@umn.edu \\ ** Department of Automation, \\ Shanghai Jiao Tong University, Shanghai, China, \\ and \\ Center for Industrial and Applied Mathematics and ACCESS Linnaeus \\ Center, KTH Royal Institute of Technology, Stockholm, Sweden \\ alq@kth.se
}

\begin{abstract}
Reversal of the time direction in stochastic systems driven by white noise has been of central importance throughout the development of stochastic realization theory, filtering and smoothing. Similarly, in connection with certain problems in the theory of moments, the duality induced by time reversal was the key to deriving a compact parametrization and an interpretation of solutions. By combining ideas from these two lines of development we present herein a general view and a simplified account of time-reversal in stochastic models.
\end{abstract}

Keywords: Stochastic realization theory, time-reversal of stochastic models

\section{INTRODUCTION}

Time reversal of stochastic systems is central in stochastic realization theory (see, e.g., Lindquist and Picci (1979, 1985a,b, 1991), Pavon (1980), Lindquist and Pavon (1984), Michaletzky et al. (1998), Michaletzky and Ferrante (1995)), filtering (see Lindquist (1974)), smoothing (see Badawi et al. (1979a,c), Ferrante and Picci (2000)) and system identification. The principal construction is to model a stochastic process as the output of a linear system driven by a noise process which is assumed to be white in discrete time, and orthogonal-increment in continuous time. In studying the dependence between past and future of the process, it is natural to decompose the interface between past and future in a time-symmetric manner. This gives rise to systems representations of the process running in either time direction, forward or backward in time.

In a different context (see Georgiou (2007)) a duality between time-reversed stochastic models was independently introduced in order to characterize and interpret solutions to moment problems. General moment constraints correspond to state-statistics of a linear system, while the driving inputs to two time-reversed models with the same state-statistics was shown to relate via suitable all-pass dynamics. The family of power spectra for input processes that are consistent with the given statistics/moments corresponds to a matrix-ball with left and right radii related

\footnotetext{
* This research was supported by grants from AFOSR, NSF, VR, and the SSF.
}

to the prediction error variance in the two opposing timedirections, respectively.

In the present note, we combine ideas from these two lines of development into a simplified and general approach for deriving and explaining the "mechanism" behind such time-reversed dual pair of stochastic systems. In particular, we recover certain results of stochastic realization theory (Lindquist and Picci (1979), Pavon (1980), Badawi et al. (1979a)) from the 1970's using a novel procedure. For additional historical pointers see (Badawi et al., 1979b, page 506, 2nd column).

In Section 2 we explain how lifting the state-dynamics into an all-pass system allows direct correspondence between sample-paths of driving generating processes, in opposite time-directions, via causal and anti-causal mappings, respectively. In Section 3 we explain this mechanism in the context of output processes and, similarly, introduce a pair of time-opposite models. This first part of the paper focuses on time-invariant models. In Section 4 we briefly outline the corresponding results in the time-varying case. For technical details and proofs we refer to Georgiou and Lindquist (2014).

\section{STATE DYNAMICS AND ALL-PASS EXTENSION}

We first consider the stationary case. We treat both discrete-time as well as continuous-time stochastic linear state-dynamics. As usual, in discrete-time these take the form of a set of difference equations 


$$
x(t+1)=A x(t)+B u(t)
$$

where $t \in \mathbb{Z}, A \in \mathbb{R}^{n \times n}, B \in \mathbb{R}^{n \times p}, A$ has all eigenvalues in the open unit disc $\mathbb{D}=\{z|| z \mid<1\}$, and $u(t), x(t)$ are (centered) stationary vector-valued stochastic processes with $u(t)$ normalized white noise; i.e.,

$$
\mathbb{E}\left\{u(t) u(s)^{\prime}\right\}=I_{p} \delta_{t s} .
$$

The system of equations is assumed to be reachable, i.e.,

$$
\operatorname{rank}\left[B, A B, \ldots A^{n-1} B\right]=n \text {. }
$$

In continuous-time, state-dynamics take the form of a system of stochastic differential equations

$$
d x(t)=A x(t) d t+B d u(t)
$$

where, here, $x(t)$ is a stationary continuous-time vectorvalued stochastic process and $u(t)$ is a vector-valued process with orthogonal increments with the property

$$
\mathbb{E}\left\{d u d u^{\prime}\right\}=I_{p} d t .
$$

Reachability (which in this case, is equivalent to controllability) of the pair $(A, B)$ is also assumed throughout and the condition for this is identical to the one for discretetime given above (as is well known). In continuous time, stability of the system of equations is equivalent to $A$ having only eigenvalues with negative real part.

In either case, discrete-time or continuous-time, it is possible to define an output equation so that the overall system is all-pass. This is done next.

\subsection{All-pass extension in discrete-time}

Consider the discrete-time Lyapunov equation

$$
P=A P A^{\prime}+B B^{\prime} \text {. }
$$

Since $A$ has all eigenvalues inside the unit disc of the complex plane and (3) holds, (6) has as solution a matrix $P$ which is positive definite. The state transformation

$$
\xi=P^{-\frac{1}{2}} x
$$

and

$$
F=P^{-\frac{1}{2}} A P^{\frac{1}{2}}, G=P^{-\frac{1}{2}} B,
$$

brings (1) into

$$
\xi(t+1)=F \xi(t)+G u(t) .
$$

For this new system, the corresponding Lyapunov equation $X=F X F^{\prime}+G G^{\prime}$ has $I_{n}$ as solution, where $I_{n}$ denotes the $(n \times n)$ identity matrix. This fact, namely, that

$$
I_{n}=F F^{\prime}+G G^{\prime}
$$

implies that this $[F, G]$ can be embedded as part of an orthogonal matrix

$$
U=\left[\begin{array}{ll}
F & G \\
H & J
\end{array}\right]
$$

i.e., a matrix such that $U U^{\prime}=U^{\prime} U=I_{n+p}$.

Define the transfer function

$$
\mathbf{U}(z):=H\left(z I_{n}-F\right)^{-1} G+J
$$

corresponding to

$$
\begin{aligned}
\xi(t+1) & =F \xi(t)+G u(t) \\
\bar{u}(t) & =H \xi(t)+J u(t) .
\end{aligned}
$$

This is also the transfer function of

$$
\begin{aligned}
x(t+1) & =A x(t)+B u(t) \\
\bar{u}(t) & =\bar{B}^{\prime} x(t)+J u(t),
\end{aligned}
$$

where $\bar{B}:=P^{-\frac{1}{2}} H^{\prime}$, since the two systems are related by a similarity transformation. Hence,

$$
\mathbf{U}(z)=\bar{B}^{\prime}\left(z I_{n}-A\right)^{-1} B+J .
$$

We claim that $\mathbf{U}(z)$ is a stable all-pass transfer function (with respect to the unit disc), i.e., that $\mathbf{U}(z)$ is a transfer function of a stable system and that

$$
\mathbf{U}(z) \mathbf{U}\left(z^{-1}\right)^{\prime}=\mathbf{U}\left(z^{-1}\right)^{\prime} \mathbf{U}(z)=I_{p} .
$$

The latter claim is immediate after we observe that, since $U^{\prime} U=I_{n+p}$,

$$
U^{\prime}\left[\begin{array}{c}
\xi(t+1) \\
\bar{u}(t)
\end{array}\right]=\left[\begin{array}{l}
\xi(t) \\
u(t)
\end{array}\right]
$$

and hence,

$$
\begin{aligned}
& \xi(t)=F^{\prime} \xi(t+1)+H^{\prime} \bar{u}(t) \\
& u(t)=G^{\prime} \xi(t+1)+J^{\prime} \bar{u}(t)
\end{aligned}
$$

or, equivalently,

$$
\begin{aligned}
& x(t)=P A^{\prime} P^{-1} x(t+1)+P^{\frac{1}{2}} H^{\prime} u(t) \\
& u(t)=B^{\prime} P^{-1} x(t+1)+J^{\prime} \bar{u}(t) .
\end{aligned}
$$

Setting

(18) can be written

$$
\bar{x}(t):=P^{-1} x(t+1),
$$

$$
\begin{aligned}
\bar{x}(t-1) & =A^{\prime} \bar{x}(t)+\bar{B} \bar{u}(t) \\
u(t) & =B^{\prime} \bar{x}(t)+J^{\prime} \bar{u}(t)
\end{aligned}
$$

with transfer function

$$
\mathbf{U}\left(z^{-1}\right)^{\prime}=B^{\prime}\left(z^{-1} I_{n}-A^{\prime}\right)^{-1} \bar{B}+J^{\prime} .
$$

Either of the above systems (17) or (20) inverts the dynamical relation $u \rightarrow \bar{u}$ in (14) or (13) by reversing time.

\subsection{All-pass extension in continuous-time}

Consider the continuous-time Lyapunov equation

$$
A P+P A^{\prime}+B B^{\prime}=0 .
$$

Since $A$ has all its eigenvalues in the left half of the complex plane and since (3) holds, (22) has as solution a positive definite matrix $P$. Once again, applying (7-8), the system in (4) becomes

$$
d \xi(t)=F \xi(t) d t+G d u(t) .
$$

We now seek a completion by adding an output equation

$$
d \bar{u}(t)=H \xi(t) d t+J d u(t)
$$

so that the transfer function

$$
\mathbf{U}(s):=H\left(s I_{n}-F\right)^{-1} G+J
$$

is all-pass (with respect to the imaginary axis), i.e.,

$$
\mathbf{U}(s) \mathbf{U}(-s)^{\prime}=\mathbf{U}(-s)^{\prime} \mathbf{U}(s)=I_{p} .
$$


For this new system, the corresponding Lyapunov equation has as solution the identity matrix and hence,

$$
F+F^{\prime}+G G^{\prime}=0 .
$$

It can be shown that $(25)$ holds provided $J J^{\prime}=I_{p}$ and $H=-J G^{\prime}$. Thus, we take

$$
\begin{aligned}
& J=I_{p} \\
& H=-G^{\prime},
\end{aligned}
$$

and obtain the forward dynamics

$$
\begin{aligned}
d \xi(t) & =F \xi(t) d t+G d u(t) \\
d \bar{u}(t) & =-G^{\prime} \xi(t) d t+d u(t) .
\end{aligned}
$$

Substituting $F=-F^{\prime}-G G^{\prime}$ from (26) into (27a) we obtain the reverse-time dynamics

$$
\begin{aligned}
& d \xi(t)=-F^{\prime} \xi(t) d t+G d \bar{u}(t) \\
& d u(t)=G^{\prime} \xi(t) d t+d \bar{u}(t) .
\end{aligned}
$$

Now defining

$$
\bar{x}(t):=P^{-1} x(t)
$$

and using (7) and (8), (28) becomes

$$
\begin{aligned}
& d \bar{x}(t)=-A^{\prime} \bar{x}(t) d t+\bar{B} d \bar{u}(t) \\
& d u(t)=B^{\prime} \bar{x}(t) d t+d \bar{u}(t),
\end{aligned}
$$

with transfer function

$$
\mathbf{U}(-s)^{\prime}=I_{p}+B^{\prime}\left(s I_{n}+A^{\prime}\right)^{-1} \bar{B},
$$

where

$$
\bar{B}:=P^{-1} B .
$$

Furthermore, the forward dynamics (27) can be expressed in the form

$$
\begin{gathered}
d x(t)=A x(t) d t+B d u(t) \\
d \bar{u}(t)=-\bar{B}^{\prime} x(t) d t+d u(t)
\end{gathered}
$$

with transfer function

$$
\mathbf{U}(s)=I_{p}-\bar{B}^{\prime}\left(s I_{n}-A\right)^{-1} B .
$$

\section{TIME-REVERSAL OF STATIONARY LINEAR STOCHASTIC SYSTEMS}

The development so far allows us to draw a connection between two linear stochastic systems having the same output and driven by a pair of arbitrary, but dual, stationary processes $u(t)$ and $\bar{u}(t)$, one evolving forward in time and one evolving backward in time. When one of these two processes is white noise (or an orthogonal increment process in continuous-time), then so is the other. For this special case we recover results of Lindquist and Picci (1979) and Pavon (1980) in stochastic realization theory.

\subsection{Time-reversal of discrete-time stochastic systems}

Consider a stochastic linear system

$$
\begin{aligned}
x(t+1) & =A x(t)+B u(t) \\
y(t) & =C x(t)+D u(t)
\end{aligned}
$$

with an $m$-dimensional output process $y$, and $x, u, A, B$ are defined as in Section 2.1. All processes are stationary and the system can be thought of as evolving forward in time from the remote past $(t=-\infty)$. In particular,

$$
\left(\begin{array}{c}
x(t+1) \\
y(t)
\end{array}\right) \text { is } \mathcal{F}_{t}^{u} \text {-measurable }
$$

for all $t \in \mathbb{Z}$, where $\mathcal{F}_{t}^{u}$ is the $\sigma$-algebra generated by $\{u(s) \mid s \leq t\}$. Next we construct a stochastic system

$$
\begin{aligned}
\bar{x}(t-1) & =A^{\prime} \bar{x}(t)+\bar{B} \bar{u}(t) \\
y(t) & =\bar{C} \bar{x}(t)+\bar{D} \bar{u}(t),
\end{aligned}
$$

which evolves backward in time from the remote future $(t=\infty)$, and for which

$$
\left(\begin{array}{c}
\bar{x}(t-1) \\
y(t)
\end{array}\right) \text { is } \overline{\mathcal{F}}_{t}^{\bar{u}} \text {-measurable }
$$

for all $t \in \mathbb{Z}$, where $\overline{\mathcal{F}}_{t}^{\bar{u}}$ is the $\sigma$-algebra generated by $\{\bar{u}(s) \mid s \geq t\}$. The processes $\bar{x}, x, \bar{u}, u$ relate as in the previous section. More specifically, it can be shown that

$$
\bar{u}(t) \text { is } \mathcal{F}_{t}^{u} \text {-measurable }
$$

while

$$
u(t) \text { is } \overline{\mathcal{F}}_{t}^{\bar{u}} \text {-measurable }
$$

for all $t, \bar{u}$ is a white noise process and $\mathbb{E}\left\{\bar{x}(t) \bar{u}(s)^{\prime}\right\}=0$ for all $s \leq t$.

In fact, the all-pass extension (14) of (35a) yields

$$
\bar{u}(t)=\bar{B}^{\prime} x(t)+J u(t)
$$

It follows from (20b) that (37) can be inverted to yield

$$
u(t)=B^{\prime} \bar{x}(t)+J^{\prime} \bar{u}(t),
$$

where $\bar{x}(t)=P^{-1} x(t+1)$, and that we have the reversetime recursion

$$
\bar{x}(t-1)=A^{\prime} \bar{x}(t)+\bar{B} \bar{u}(t) .
$$

Then inserting (38) and

$$
x(t)=P \bar{x}(t-1)=P A^{\prime} \bar{x}(t)+P \bar{B} \bar{u}(t)
$$

into (35b), we obtain

$$
y(t)=\bar{C} \bar{x}(t)+\bar{D} \bar{u}(t),
$$

where $\bar{D}:=C P \bar{B}+D J^{\prime}$ and

$$
\bar{C}:=C P A^{\prime}+D B^{\prime} .
$$

Then, (39) is precisely what we wanted to establish.

Moreover, the transfer functions

$$
\mathbf{W}(z)=C\left(z I_{n}-A\right)^{-1} B+D
$$

of (35) and

$$
\overline{\mathbf{W}}(z)=\bar{C}\left(z^{-1} I_{n}-A^{\prime}\right)^{-1} \bar{B}+\bar{D}
$$

of (36) satisfy

$$
\mathbf{W}(z)=\overline{\mathbf{W}}(z) \mathbf{U}(z) .
$$

In the context of stochastic realization theory, discussed next, $\mathbf{U}(z)$ is called structural function (Lindquist and Picci (1985b, 1991); Lindquist and Pavon (1984)).

\subsection{Time-reversal of continuous-time stochastic systems}

We now turn to the continuous-time case. Let

$$
\begin{aligned}
& d x=A x d t+B d u \\
& d y=C x d t+D d u
\end{aligned}
$$


be a stochastic system with $x, u, A, B$ as in Section 2.2 , evolving forward in time from the remote past $(t=-\infty)$. All processes have stationary increments and

$$
\left(\begin{array}{l}
x(t) \\
y(t)
\end{array}\right) \text { is } \mathcal{F}_{t}^{u} \text {-measurable }
$$

for all $t \in \mathbb{R}$, where $\mathcal{F}_{t}^{u}$ is the $\sigma$-algebra generated by $\{u(s) \mid s \leq t\}$.

The all-pass extension of Section 2.2 yields

$$
d \bar{u}=d u-\bar{B}^{\prime} x d t
$$

as well as the reverse-time relation

$$
\begin{aligned}
d \bar{x} & =-A^{\prime} \bar{x} d t+\bar{B} d \bar{u} \\
d u & =B^{\prime} \bar{x} d t+d \bar{u},
\end{aligned}
$$

where $\bar{x}(t)=P^{-1} x(t)$. Inserting (46b) into

$$
d y=C P \bar{x} d t+D d u
$$

yields

$$
d y=\bar{C} \bar{x} d t+D d \bar{u}
$$

where

$$
\bar{C}=C P+D B^{\prime} .
$$

Thus, the reverse-time system is

$$
\begin{aligned}
& d \bar{x}=-A^{\prime} \bar{x} d t+\bar{B} d \bar{u} \\
& d y=\bar{C} \bar{x} d t+D d \bar{u} .
\end{aligned}
$$

From this, we deduce that

$$
\left(\begin{array}{l}
\bar{x}(t) \\
y(t)
\end{array}\right) \text { is } \overline{\mathcal{F}}_{t}^{\bar{u}} \text {-measurable }
$$

for all $t \in \mathbb{R}$. We also note that the transfer function

$$
\mathbf{W}(s)=C\left(s I_{n}-A\right)^{-1} B+D
$$

of (44) and the transfer function

$$
\overline{\mathbf{W}}(s)=\bar{C}\left(s I_{n}+A^{\prime}\right)^{-1} \bar{B}+D
$$

of (48) also satisfy

as in discrete-time.

$$
\mathbf{W}(s)=\overline{\mathbf{W}}(s) \mathbf{U}(s)
$$

\section{THE NON-STATIONARY CASE}

We briefly outline an extension of the above results to the case of linear time-varying systems. A detailed exposition and proofs are given in Georgiou and Lindquist (2014).

\subsection{The discrete-time case}

Consider the non-stationary state dynamics

$$
x(t+1)=A(t) x(t)+B(t) u(t)
$$

on a finite time-window $[0, T]$. The state covariance matrix $P(t):=\mathbb{E}\left\{x(t) x(t)^{\prime}\right\}$ satisfies the Lyapunov difference equation

$$
P(t+1)=A(t) P(t) A(t)^{\prime}+B(t) B(t)^{\prime} .
$$

The state transformation

$$
\xi(t)=P(t)^{-\frac{1}{2}} x(t)
$$

brings the system (49) into

$$
\xi(t+1)=F(t) \xi(t)+G(t) u(t)
$$

where now $\mathbb{E}\left\{\xi(t) \xi(t)^{\prime}\right\}=I_{n}$ for all $t$,

$$
\begin{aligned}
& F(t)=P(t+1)^{-\frac{1}{2}} A(t) P(t)^{\frac{1}{2}}, \\
& G(t)=P(t+1)^{-\frac{1}{2}} B,
\end{aligned}
$$

and

$$
F(t) F(t)^{\prime}+G(t) G(t)^{\prime}=I_{n}
$$

This allows us to embed $[F, G]$ into a time-varying orthogonal matrix

$$
U(t)=\left[\begin{array}{ll}
F(t) & G(t) \\
H(t) & J(t)
\end{array}\right]
$$

which extends (52) to

$$
\begin{aligned}
\xi(t+1) & =F(t) \xi(t)+G(t) u(t) \\
\bar{u}(t) & =H(t) \xi(t)+J(t) u(t) .
\end{aligned}
$$

Now, premultiplying (56) by $U(t)^{\prime}$, we obtain the dual stochastic system

$$
\begin{aligned}
\xi(t) & =F(t)^{\prime} \xi(t+1)+H(t)^{\prime} \bar{u}(t) \\
u(t) & =G(t)^{\prime} \xi(t+1)+J(t)^{\prime} \bar{u}(t),
\end{aligned}
$$

which runs backwards in time.

Using the transformation (51), (56) yields the forward representation

$$
\begin{aligned}
x(t+1) & =A(t) x(t)+B(t) u(t) \\
\bar{u}(t) & =\bar{B}(t)^{\prime} x(t)+J(t) u(t),
\end{aligned}
$$

where $\bar{B}(t):=P(t)^{-\frac{1}{2}} H(t)^{\prime}$. Likewise (57) and

$$
\bar{x}(t)=P(t+1)^{-1} x(t+1),
$$

yield the backward representation

$$
\begin{aligned}
\bar{x}(t-1) & =A(t)^{\prime} \bar{x}(t)+\bar{B}(t) \bar{u}(t) \\
u(t) & =B(t)^{\prime} \bar{x}(t)+J(t)^{\prime} \bar{u}(t) .
\end{aligned}
$$

We are now in a position to derive a backward version of a non-stationary stochastic system

$$
\begin{aligned}
x(t+1) & =A(t) x(t)+B(t) u(t) \\
y(t) & =C(t) x(t)+D(t) u(t) .
\end{aligned}
$$

In fact, inserting (59) and (60a) into (61b) yields

$$
y(t)=\bar{C} \bar{x}(t)+\bar{D} \bar{u}(t)
$$

where

$$
\begin{aligned}
& \bar{C}=C(t) P(t) A(t)^{\prime}+D(t) B(t)^{\prime} \\
& \bar{D}=C(t) P(t) \bar{B}(t)+D(t) J(t)^{\prime} .
\end{aligned}
$$

Finally, this gives the backward system

$$
\begin{aligned}
\bar{x}(t-1) & =A(t)^{\prime} \bar{x}(t)+\bar{B}(t) \bar{u}(t) \\
y(t) & =\bar{C}(t) \bar{x}(t)+\bar{D}(t) \bar{u}(t) .
\end{aligned}
$$

Remark 1. When considered on the doubly infinite time axis, equation (56) defines an isometry. Indeed, assuming that the input is square-summable, the fact that $U(t)$ is unitary for all $t$ directly implies that

$$
\sum_{-\infty}^{N}\|\bar{u}\|^{2}+\|\xi(t+1)\|^{2}=\sum_{-\infty}^{N}\|u(t)\|^{2} .
$$

Then, $\xi(t) \rightarrow 0$ as $t \rightarrow \infty$, provided the transition matrix $\Phi(t, s)$ of (56) tends to 0 as $s \rightarrow-\infty$. It follows that

$$
\sum_{t=-\infty}^{\infty}\|\bar{u}(t)\|^{2}=\sum_{t=-\infty}^{\infty}\|u(t)\|^{2} .
$$




\subsection{The continuous-time case}

The covariance matrix function $P(t):=\mathbb{E}\left\{x(t) x(t)^{\prime}\right\}$ of the time-varying state representation

$$
d x=A(t) x(t) d t+B(t) d u
$$

satisfies the matrix-valued differential equation

$$
\dot{P}=A(t) P(t)+P(t) A(t)^{\prime}+B(t) B(t)^{\prime} .
$$

A unitary extension of (65) is somewhat more complicated than in the discrete time case. Differentiating

$$
\xi(t)=P(t)^{-\frac{1}{2}} x(t)
$$

we obtain

$$
d \xi=F(t) \xi(t) d t+G(t) d u
$$

where $F, G$ now depend on the derivative of $P$ (see Georgiou and Lindquist (2014) for precise expressions) and once again satisfy

$$
F(t)+F(t)^{\prime}+G(t) G(t)^{\prime}=0 .
$$

Using (69) to eliminate $F$ in (68), we obtain

$$
d \xi=-F(t)^{\prime} \xi(t) d t+G(t) d \bar{u}
$$

where

$$
d \bar{u}=d u-G(t)^{\prime} \xi(t) d t .
$$

Equations (68) and (71) correspond to the forward in time stochastic system

$$
\begin{aligned}
d x & =A(t) x(t) d t+B(t) d u \\
d \bar{u} & =-\bar{B}(t)^{\prime} x(t) d t+d u,
\end{aligned}
$$

where $\bar{B}(t):=P(t)^{-1} B(t)$. Moreover, setting $\bar{x}(t)=$ $P(t)^{\frac{1}{2}} \xi(t)$, we obtain

$$
\begin{aligned}
& d \bar{x}=-A(t)^{\prime} \bar{x}(t) d t+\bar{B}(t) d \bar{u} \\
& d u=B(t)^{\prime} \bar{x}(t) d t+d \bar{u}
\end{aligned}
$$

which constitutes the corresponding backward stochastic system.

Next we derive the backward stochastic realization corresponding to

$$
\begin{aligned}
& d x=A(t) x(t) d t+B(t) d u \\
& d y=C(t) x(t) d t+D(t) d u
\end{aligned}
$$

defined on the finite interval $[0, T]$. To this end, apply the transformation $\bar{x}(t)=P(t)^{-1} x(t)$ together with (73b) to (74b) to obtain

$$
d y=\bar{C}(t) \bar{x}(t)+D(t) d \bar{u},
$$

where

$$
\bar{C}(t)=C(t) P(t)+D(t) B(t) .
$$

This together with (73a) yields the backward system corresponding to (74), namely

$$
\begin{aligned}
& d \bar{x}=-A(t)^{\prime} \bar{x}(t) d t+\bar{B}(t) d \bar{u} \\
& d y=\bar{C}(t) \bar{x}(t) d t+D(t) d \bar{u} .
\end{aligned}
$$

Technical details and proofs are given in Georgiou and Lindquist (2014). The backward realization (76) was derived in Badawi et al. (1979c), but in a cumbersome way, requiring the proof that $\bar{u}(t)$ is a normalized process with orthogonal increments to be suppressed. What is new here is the unitary map between $u$ and $\bar{u}$, which makes the analysis much simpler and more transparent (see Georgiou and Lindquist (2014)).

\section{CONCLUDING REMARKS}

Dual time-reversed stochastic systems have been employed to model, in different time-directions, Brownian or Schrödinger bridges (see Pavon and Wakolbinger (1991), Dai Pra and Pavon (1990)), a subject which is related to reciprocal processes (Jamison (1974), Krener (1986), Levy et al. (1990), Dai Pra (1991)). The topic of time reversibility has also been central to thermodynamics, and in recent years studies have sought to elucidate its relation to systems theory (see Haddad et al. (2008, 2009)).

The thesis of the present work is that under mild assumptions on a stochastic process, any model that consists of a linear stable dynamical system driven by an appropriate input process can be reversed in time. In fact, a reverse-time dual system along with the corresponding input process can be obtained via an all-pass extension of the state equation. The correspondence between the two input processes can be expressed in terms of each other by a causal and an anti-causal map, respectively. The novel element in our approach is the unitary imbedding.

\section{REFERENCES}

Badawi, F., Lindquist, A., and Pavon, M. (1979a). On the Mayne-Fraser smoothing formula and stochastic realization theory for nonstationary linear stochastic systems. In Decision and Control including the Symposium on Adaptive Processes, 1979 18th IEEE Conference on, volume 18, 505-510. IEEE.

Badawi, F., Lindquist, A., and Pavon, M. (1979b). On the mayne-fraser smoothing formula and stochastic realization theory for nonstationary linear stochastic systems. In Decision and Control including the Symposium on Adaptive Processes, 1979 18th IEEE Conference on, volume 18, 505-510. IEEE.

Badawi, F.A., Lindquist, A., and Pavon, M. (1979c). A stochastic realization approach to the smoothing problem. IEEE Trans. Automat. Control, 24(6), 878888.

Dai Pra, P. (1991). A stochastic control approach to reciprocal diffusion processes. Applied mathematics and Optimization, 23(1), 313-329.

Dai Pra, P. and Pavon, M. (1990). On the Markov processes of Schrödinger, the Feynman-Kac formula and stochastic control. In Realization and Modelling in System Theory, 497-504. Springer.

Ferrante, A. and Picci, G. (2000). Minimal realization and dynamic properties of optimal smoothers. Automatic Control, IEEE Transactions on, 45(11), 2028-2046.

Georgiou, T. and Lindquist, A. (2014). Reversing time in stochastic models, redux. arxiv report.

Georgiou, T.T. (2007). The Carathéodory-FejérPisarenko decomposition and its multivariable counterpart. Automatic Control, IEEE Transactions on, 52(2), 212-228.

Haddad, W.M., Chellaboina, V., and Nersesov, S.G. (2008). Time-reversal symmetry, poincaré recurrence, 
irreversibility, and the entropic arrow of time: From mechanics to system thermodynamics. Nonlinear Analysis: Real World Applications, 9(2), 250-271.

Haddad, W.M., Chellaboina, V., and Nersesov, S.G. (2009). Thermodynamics: A dynamical systems approach. Princeton University Press.

Jamison, B. (1974). Reciprocal processes. Probability Theory and Related Fields, 30(1), 65-86.

Krener, A. (1986). Reciprocal processes and the stochastic realization problem for acausal systems. In C.I. Byrnes and A. Lindquist (eds.), Modelling, Identification and Robust Control, 197-211. North-Holland, Amsterdam.

Levy, B.C., Frezza, R., and Krener, A.J. (1990). Modeling and estimation of discrete-time gaussian reciprocal processes. Automatic Control, IEEE Transactions on, 35(9), 1013-1023.

Lindquist, A. (1974). A new algorithm for optimal filtering of discrete-time stationary processes. SIAM J. Control, $12,736-746$.

Lindquist, A. and Pavon, M. (1984). On the structure of state-space models for discrete-time stochastic vector processes. IEEE Trans. Automat. Control, 29(5), 418432.

Lindquist, A. and Picci, G. (1979). On the stochastic realization problem. SIAM J. Control Optim., 17(3),
$365-389$.

Lindquist, A. and Picci, G. (1985a). Forward and backward semimartingale models for Gaussian processes with stationary increments. Stochastics, 15(1), 1-50.

Lindquist, A. and Picci, G. (1985b). Realization theory for multivariate stationary Gaussian processes. SIAM J. Control Optim., 23(6), 809-857.

Lindquist, A. and Picci, G. (1991). A geometric approach to modelling and estimation of linear stochastic systems. J. Math. Systems Estim. Control, 1(3), 241-333.

Michaletzky, G., Bokor, J., and Várlaki, P. (1998). Representability of stochastic systems. Akadémiai Kiadó, Budapest.

Michaletzky, G. and Ferrante, A. (1995). Splitting subspaces and acausal spectral factors. J. Math. Systems Estim. Control, 5(3), 1-26.

Pavon, M. (1980). Stochastic realization and invariant directions of the matrix Riccati equation. SIAM Journal on Control and Optimization, 18(2), 155-180.

Pavon, M. and Wakolbinger, A. (1991). On free energy, stochastic control, and Schrödinger processes. In Modeling, Estimation and Control of Systems with Uncertainty, 334-348. Springer. 